\title{
The Leading Finite Type Coefficients of the Links-Gould Polynomial of a Link
}

Atsushi IshiI

Institute of Mathematics, University of Tsukuba Tsukuba, Ibaraki 305-8571, Japan

e-mail : aishii@math.tsukuba.ac.jp

ABSTRACT. We show that the Links-Gould polynomial of a link has finite type coefficients in a multivariate series expansion, and express the leading coefficients in terms of the linking numbers of a link.

\section{Introduction}

The Links-Gould polynomial [11] is a quantum invariant which is derived from the one-parameter family of four dimensional representations of the quantum superalgebra $U_{q}[g l(2 \mid 1)]$. D. De Wit, L. H. Kauffman and J. R. Links [4] gave an explicit form of the $R$-matrix for the invariant, and showed that it is a powerful invariant through its evaluation. The invariant is complete for all prime knots of up to 10 crossings [3] and for the Kanenobu knots [8], [9]. The Links-Gould polynomial is not only a powerful invariant. It is also a two-variable generalization of the Alexander-Conway polynomial [7]. In this paper, we show that the Links-Gould polynomial has finite type coefficients in a multivariate series expansion with respect to symmetrical variables, where an invariant is finite type [2], [14] if it vanishes for singular knots with finite singularities. Furthermore, we determine the leading coefficients in terms of the linking numbers of links. This result is analogous to that on the first coefficient of the Conway polynomial of a link due to Hoste [5].

For an ordered oriented $r$-component link $L=K_{1} \cup \cdots \cup K_{r}$, we denote by $\lambda_{i, j}(L)$ the linking number of $K_{i}$ and $K_{j}$. Let $\Phi$ be a graph with $r$ vertices $p_{1}, \cdots, p_{r}$ and $e(i, j)$ edges joining $p_{i}$ and $p_{j}$. We define the invariant $\Lambda_{\Phi}$ by

$$
\Lambda_{\Phi}(L)=\prod_{i<j} \lambda_{i, j}(L)^{e(i, j)}
$$

Let $\mathcal{C}_{r}$ be the set of cycle graphs with $r$ vertices $p_{1}, \cdots, p_{r}$, where a cycle graph is a connected graph which forms one cycle. Then $\sum_{\Phi \in \mathcal{C}_{r}} \Lambda_{\Phi}(L)$ is well-defined for an unordered oriented $r$-component link $L$. For example,

Received March 5, 2008; revised 7 July, 2009; accepted 6 August, 2009.

2000 Mathematics Subject Classification: 57M27.

Key words and phrases: Links-Gould polynomial, finite type invariant, Vassiliev invariant. 


$$
\begin{aligned}
\sum_{\Phi \in \mathcal{C}_{2}} \Lambda_{\Phi}(L) & =\lambda_{1,2}(L) \lambda_{2,1}(L) \\
\sum_{\Phi \in \mathcal{C}_{3}} \Lambda_{\Phi}(L)= & \lambda_{1,2}(L) \lambda_{2,3}(L) \lambda_{3,1}(L), \\
\sum_{\Phi \in \mathcal{C}_{4}} \Lambda_{\Phi}(L)= & \lambda_{1,2}(L) \lambda_{2,3}(L) \lambda_{3,4}(L) \lambda_{4,1}(L)+\lambda_{1,2}(L) \lambda_{2,4}(L) \lambda_{4,3}(L) \lambda_{3,1}(L) \\
& \quad+\lambda_{1,3}(L) \lambda_{3,2}(L) \lambda_{2,4}(L) \lambda_{4,1}(L) .
\end{aligned}
$$

We denote by $L G\left(L ; t_{0}, t_{1}\right)$ the Links-Gould polynomial of an oriented link $L$. Let $a_{0}(L)$ be the finite type invariant of type 0 , defined by

$$
a_{0}(L)= \begin{cases}1 & \text { if } L \text { is a knot } \\ 0 & \text { otherwise }\end{cases}
$$

In [7], we showed that $L G\left(L ; t_{0}, t_{1}\right) \in \mathbb{Z}\left[t_{0}^{ \pm 1}, t_{1}^{ \pm 1}\right]$ and the equalities

$$
L G\left(L ; t_{0}, 1\right)=L G\left(L ; 1, t_{1}\right)=a_{0}(L) .
$$

Then the Links-Gould polynomial is expressed in the following form:

$$
L G\left(L ; t_{0}, t_{1}\right)=a_{0}(L)+\sum_{i, j=1}^{\infty} a_{i, j}(L)\left(t_{0}-1\right)^{i}\left(t_{1}-1\right)^{j}
$$

in $\mathbb{Z}\left[\left[t_{0}-1, t_{1}-1\right]\right]$, where $a_{i, j}(L) \in \mathbb{Z}$.

Theorem 1. The coefficient $a_{i, j}(L)$ is a finite type invariant of type $i+j$. Let $r$ be the number of components of $L$. For $i+j<r$, we have $a_{i, j}(L)=0$. Furthermore, for $i+j=r$, the leading coefficients $a_{i, j}(L)$ are given by

$$
a_{i, j}(L)= \begin{cases}-\sum_{C \in \mathcal{C}_{2}} \Lambda_{C}(L) & \text { if } i=j=1, \\
-2\left(\begin{array}{c}
r-2 \\
i-1
\end{array}\right) \sum_{C \in \mathcal{C}_{r}} \Lambda_{C}(L) & \text { otherwise. }\end{cases}
$$

\section{Preliminaries}

We recall the definition of the Links-Gould polynomial. Let $V$ be a four dimensional vector space with a basis $\left\{e_{i}\right\}_{i=1}^{4}$, and let $V^{*}$ be its dual. We denote the dual basis by $\left\{e_{i}^{*}\right\}_{i=1}^{4}$ :

$$
e_{i}^{*}\left(e_{j}\right)=\delta_{j}^{i},
$$

where $\delta_{j}^{i}$ is the Kronecker symbol. We define $R: V \otimes V \rightarrow V \otimes V$ by

$$
\begin{aligned}
& t_{0} e_{11}^{11}-\left(e_{22}^{22}+e_{33}^{33}\right)+t_{1} e_{44}^{44}+\left(t_{0}-1\right)\left(e_{21}^{21}+e_{31}^{31}\right)+\left(t_{0}-1\right)\left(1-t_{1}\right) e_{41}^{41} \\
& +\left(t_{1}-1\right)\left(e_{42}^{42}+e_{43}^{43}\right)+\left(t_{0} t_{1}-1\right) e_{23}^{23}+\left(e_{41}^{14}+e_{14}^{41}\right)-t_{0}^{1 / 2} t_{1}^{1 / 2}\left(e_{32}^{23}+e_{23}^{32}\right) \\
& +t_{0}^{1 / 2}\left(e_{21}^{12}+e_{12}^{21}+e_{31}^{13}+e_{13}^{31}\right)+t_{1}^{1 / 2}\left(e_{42}^{24}+e_{24}^{42}+e_{43}^{34}+e_{34}^{43}\right) \\
& -t_{0}^{1 / 2} t_{1}^{1 / 2}\left(\left(t_{0}-1\right)\left(1-t_{1}\right)\right)^{1 / 2}\left(e_{41}^{23}+e_{23}^{41}\right)+\left(\left(t_{0}-1\right)\left(1-t_{1}\right)\right)^{1 / 2}\left(e_{41}^{32}+e_{32}^{41}\right),
\end{aligned}
$$






Figure 1:

where the map $e_{j_{1} j_{2}}^{i_{1} i_{2}}: V \otimes V \rightarrow V \otimes V$ is defined by

$$
e_{j_{1} j_{2}}^{i_{1} i_{2}}\left(e_{k_{1}} \otimes e_{k_{2}}\right)=\delta_{k_{1}}^{j_{1}} \delta_{k_{2}}^{j_{2}} e_{i_{1}} \otimes e_{i_{2}} .
$$

We define $n: V \otimes V^{*} \rightarrow \mathbb{C}$ and $\tilde{n}: V^{*} \otimes V \rightarrow \mathbb{C}$ by

$$
n\left(e_{i} \otimes e_{j}^{*}\right)=\delta_{j}^{i} \quad \text { and } \quad \tilde{n}\left(e_{i}^{*} \otimes e_{j}\right)=\mu_{i} \delta_{j}^{i},
$$

where $\left(\mu_{1}, \mu_{2}, \mu_{3}, \mu_{4}\right)=\left(t_{0},-t_{1}^{-1},-t_{0}, t_{1}^{-1}\right)$. We define $u: \mathbb{C} \rightarrow V \otimes V^{*}$ and $\tilde{u}:$ $\mathbb{C} \rightarrow V^{*} \otimes V$ by

$$
u(1)=\sum_{i=1}^{4} \mu_{i}^{-1} e_{i} \otimes e_{i}^{*} \quad \text { and } \quad \tilde{u}(1)=\sum_{i=1}^{4} e_{i}^{*} \otimes e_{i} .
$$

Any oriented tangle diagram can be expressed up to isotopy as a diagram composed from the elementary tangle diagrams shown in Figure 1. Furthermore any oriented tangle diagram can be expressed up to isotopy as a sliced diagram which is such a diagram sliced by horizontal lines such that each domain between adjacent horizontal lines has either a single crossing or a single critical point.

We associate the maps $\operatorname{id}_{V}, \operatorname{id}_{V^{*}}, R, R^{-1}, n, \tilde{n}, u$, and $\tilde{u}$ to elementary oriented tangle diagrams as described in Figure 2. Corresponding to any oriented tangle diagram $D$, we may then obtain a linear map $[D]$ as the composition of tensor products of copies of the linear maps associated to the elementary tangle diagrams within $D$. For example,

$$
[\uparrow]=\left(\mathrm{id}_{V} \otimes n\right)\left(R \otimes \mathrm{id}_{V^{*}}\right)\left(\mathrm{id}_{V} \otimes u\right) .
$$

Let $T$ be a (1,1)-tangle represented by a diagram $D_{T}$. We denote by $\widehat{T}$ the closure of $T$. The Links-Gould polynomial of the link $\widehat{T}$ is defined by the following identity:

$$
\left[D_{T}\right]=L G\left(\widehat{T} ; t_{0}, t_{1}\right) \mathrm{id}_{V}
$$

For the details we refer the reader to [4], [13].

\section{A family of singular links}

A singular link is an immersion of disjoint circles into $S^{3}$, which has transverse 
$\uparrow \begin{gathered}V \\ \uparrow \\ V\end{gathered} \operatorname{id}_{V}$
$\downarrow \begin{gathered}V_{\uparrow}^{*} \mathrm{id}_{V^{*}} \\ V^{*}\end{gathered}$

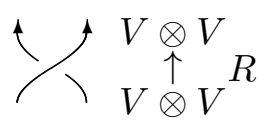


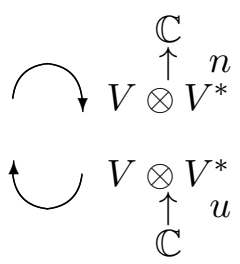<smiles>CCC[Al]O[Al](C)[Al]</smiles><smiles>CCC[Al]O[Al]</smiles>

Figure 2:



singular link

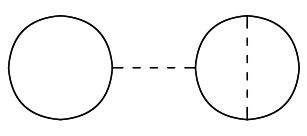

2-configuration $\alpha$

Figure 3:

double points and no other singularities. Let $v$ be an isotopy invariant for oriented links, which takes values in an abelian group. We may extend $v$ to a singular link invariant via the Vassiliev skein relation:

$$
v(\nearrow)=v(\nearrow)-v(\nearrow)
$$

A singular link invariant $v$ is called a finite type invariant of type $d$ if $v(L)=0$ for any singular link $L$ with more than $d$ singular points.

For a finite type invariant $v_{i}$ of type $d_{i}(i=1,2)$, the product $v_{1} v_{2}$ is a finite type invariant of type $d_{1}+d_{2}$. We remark that $\left(v_{1} v_{2}\right)(L)$ is defined as follows: If $L$ has a singular point, we use the Vassiliev skein relation. Then we use the equality $\left(v_{1} v_{2}\right)(L)=v_{1}(L) v_{2}(L)$ for an oriented singular link $L$ without singular points. For a graph $\Phi$ with $d$ edges, the invariant $\Lambda_{\Phi}$ is a finite type invariant of type $d$.

A $d$-configuration is $d$ pairs of $2 d$ points on disjoint circles. A singular link with $d$ singular points respects a $d$-configuration if each singular point represents a pair of the $d$-configuration. In Figure 3 , we give an example of a $d$-configuration and a singular link respecting it. For any $d$-configuration, there exists a singular link respecting it. The configuration given in Figure 4 is called inadmissible. A configuration is called admissible if it is not inadmissible. For the details we refer the reader to [1], [2].

For a $d$-configuration $\alpha$ with $r$ circles $S_{1}, \cdots, S_{r}$, we define a graph $\Phi(\alpha)$ with $r$ vertices $p_{1}, \cdots, p_{r}$ and $d$ edges by deforming the circle $S_{k}$ into the vertex $p_{k}$ and a chord joining $S_{i}$ to $S_{j}$ into an edge joining $p_{i}$ to $p_{j}$ (see Figure 3). Let $\alpha_{i}^{d, r} ; 1 \leq i \leq f_{d, r}$ be a list of the all distinct admissible $d$-configurations with $r$ circles $S_{1}, \cdots, S_{r}$. We set 


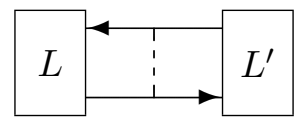

Figure 4:

$\mathcal{A}^{d, r}:=\left\{\alpha_{i}^{d, r} \mid 1 \leq i \leq f_{d, r}\right\}$,

$\mathcal{A}_{\infty}^{d, r}:=\left\{\alpha \in \mathcal{A}^{d, r} \mid \alpha\right.$ is disconnected $\}$,

$\mathcal{A}_{0}^{d, r}:=\left\{\alpha \in \mathcal{A}^{d, r} \mid \alpha\right.$ is a spanning tree $\}$,

$\mathcal{A}_{l}^{d, r}:=\left\{\alpha \in \mathcal{A}^{d, r} \mid \alpha\right.$ is a connected graph with one cycle of length $\left.l\right\}$,

for $l \geq 2$. We remark that $\mathcal{A}_{0}^{d, r}=\emptyset$ if $d \neq r-1$ and that $\mathcal{A}_{l}^{d, r}=\emptyset$ if $d \neq r$. For example, we have

$$
\begin{aligned}
& \mathcal{A}^{0,2}=\mathcal{A}_{\infty}^{0,2}=\left\{\begin{array}{c}
\cdot \\
\cdot
\end{array}\right\}, \quad \mathcal{A}^{1,2}=\mathcal{A}_{0}^{1,2}=\{!\}, \\
& \mathcal{A}^{2,2}=\mathcal{A}_{\infty}^{2,2} \cup \mathcal{A}_{2}^{2,2}=\left\{\begin{array}{cc}
\infty & \cdot \\
\bullet & \infty
\end{array}\right\} \cup\{\bigcirc\} \\
& \mathcal{A}^{0,3}=\mathcal{A}_{\infty}^{0,3}, \quad \mathcal{A}^{1,3}=\mathcal{A}_{\infty}^{1,3}, \\
& \mathcal{A}^{2,3}=\mathcal{A}_{\infty}^{2,3} \cup \mathcal{A}_{0}^{2,3}, \quad \mathcal{A}_{0}^{2,3}=\{\lfloor, \Lambda, \backslash\}, \\
& \mathcal{A}^{3,3}=\mathcal{A}_{\infty}^{3,3} \cup \mathcal{A}_{2}^{3,3} \cup \mathcal{A}_{3}^{3,3},
\end{aligned}
$$

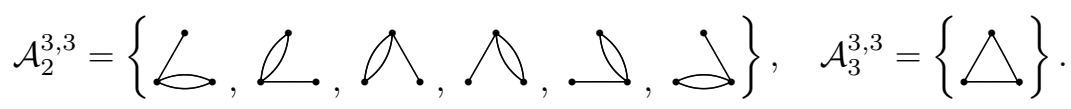

Let $\theta_{1}$ and $\theta_{2}(n)$ be the singular links as shown in Figure 5. For $\alpha \in \mathcal{A}_{\infty}^{d, r}$, we choose an ordered split singular link $M(\alpha)$ respecting $\alpha$. For $\alpha \in \mathcal{A}_{0}^{d, r}$, let $M(\alpha)$ be the connected sum of $d$ copies of $\theta_{1}$ such that $M(\alpha)$ respects $\alpha$. For $\alpha \in \mathcal{A}_{l}^{d, r}$, let $M(\alpha)$ be the connected sum of $\theta_{2}(l)$ and $d-l$ copies of $\theta_{1}$ such that $M(\alpha)$ respects $\alpha$.

We set

$$
\begin{array}{ll}
\mathcal{M}^{d, r}:=\left\{M(\alpha) \mid \alpha \in \mathcal{A}^{d, r}\right\}, & \mathcal{M}_{\infty}^{d, r}:=\left\{M(\alpha) \mid \alpha \in \mathcal{A}_{\infty}^{d, r}\right\}, \\
\mathcal{M}_{0}^{d, r}:=\left\{M(\alpha) \mid \alpha \in \mathcal{A}_{0}^{d, r}\right\}, & \mathcal{M}_{l}^{d, r}:=\left\{M(\alpha) \mid \alpha \in \mathcal{A}_{l}^{d, r}\right\}, \\
\widetilde{\mathcal{M}}^{d, r}:=\mathcal{M}^{0, r} \cup \cdots \cup \mathcal{M}^{d, r}, & \widetilde{\mathcal{M}}_{\infty}^{d, r}:=\mathcal{M}_{\infty}^{0, r} \cup \cdots \cup \mathcal{M}_{\infty}^{d, r} .
\end{array}
$$



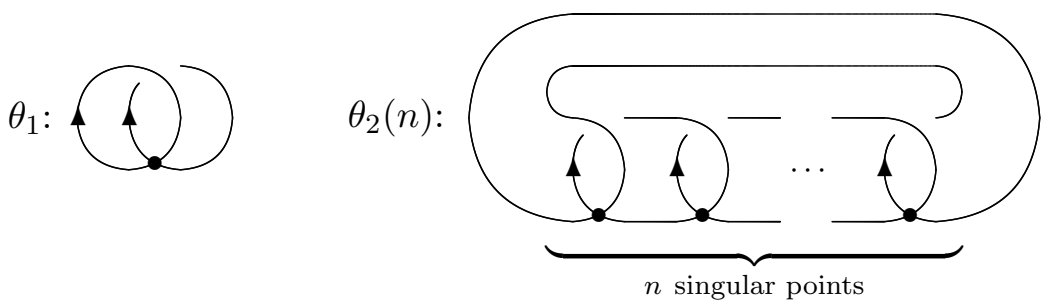

Figure 5:

Then

$$
\widetilde{\mathcal{M}}^{d, r}= \begin{cases}\widetilde{\mathcal{M}}_{\infty}^{d, r} & \text { if } d \leq r-2, \\ \widetilde{\mathcal{M}}_{\infty}^{r-1, r} \cup \mathcal{M}_{0}^{r-1, r} & \text { if } d=r-1, \\ \widetilde{\mathcal{M}}_{\infty}^{r, r} \cup \mathcal{M}_{0}^{r-1, r} \cup \mathcal{M}_{2}^{r, r} \cup \cdots \cup \mathcal{M}_{r}^{r, r} & \text { if } d=r .\end{cases}
$$

Put $\Phi(M(\alpha)):=\Phi(\alpha)$ for $\alpha \in \mathcal{A}^{d, r}$. We have the following lemma by direct calculation.

Lemma 2. Let $C$ be a cycle graph of length $r \geq 3$. For $M \in \widetilde{\mathcal{M}}^{r, r}$, we have

$$
\Lambda_{C}(M)= \begin{cases}1 & \text { if } \Phi(M)=C, \\ 0 & \text { otherwise }\end{cases}
$$

\section{Proof of theorem 1}

The Links-Gould polynomial satisfies the following skein relations [6]:



$$
\begin{aligned}
& L G(\vec{S})-L G(\bigvee)=(1-s) L G(\Omega)+(s-1) L G(\precsim) \text {, }
\end{aligned}
$$

where $s=-\left(t_{0}-1\right)\left(t_{1}-1\right)$. Put $T_{i}=t_{i}-1$ for $i=0,1$. By using these skein relations, we obtain the following skein relation:

(1) $L G(S \mathrm{SR})=\left(T_{0}+T_{1}-T_{0} T_{1}\right) L G(S)+\left(T_{0} T_{1}^{2}+T_{0}^{2} T_{1}\right) L G(\leadsto)$

$$
\left.-\left(2 T_{0} T_{1}+T_{0} T_{1}^{2}+T_{0}^{2} T_{1}+T_{0}^{2} T_{1}^{2}\right) L G() \circlearrowright\right) .
$$

We denote by $L_{1} \# L_{2}$ a connected sum of $L_{1}$ and $L_{2}$. Then we have

$$
L G\left(L_{1} \# L_{2}\right)=L G\left(L_{1}\right) L G\left(L_{2}\right) .
$$


Lemma 3. For $n \geq 2$, we have

$$
\begin{aligned}
L G\left(\theta_{1}\right) & =-T_{0} T_{1}, \\
L G\left(\theta_{2}(n)\right) & =-2\left(T_{0}+T_{1}\right)^{n-2} T_{0} T_{1}+\sum_{i+j>n} a_{i, j}\left(\theta_{2}(n)\right) T_{0}^{i} T_{1}^{j} .
\end{aligned}
$$

Proof. By the definition of the Links-Gould polynomial, we have

$$
\begin{aligned}
L G\left(\theta_{1}\right) & =-T_{0} T_{1}, \\
L G\left(\theta_{2}(1)\right)= & 0, \\
L G\left(\theta_{2}(2)\right)= & -2 T_{0} T_{1}-T_{0} T_{1}^{2}-T_{0}^{2} T_{1}-T_{0}^{2} T_{1}^{2}, \\
L G\left(\theta_{2}(3)\right)= & -2\left(T_{0}+T_{1}\right) T_{0} T_{1}+\sum_{i+j>3} a_{i, j}\left(\theta_{2}(3)\right) T_{0}^{i} T_{1}^{j}, \\
L G\left(\theta_{2}(n)\right)= & \left(T_{0}+T_{1}-T_{0} T_{1}\right) L G\left(\theta_{2}(n-1)\right)+\left(T_{0} T_{1}^{2}+T_{0}^{2} T_{1}\right) L G\left(\theta_{2}(n-2)\right) \\
& +\left(2+T_{0}+T_{1}+T_{0} T_{1}\right)(-1)^{n-1} T_{0}^{n-1} T_{1}^{n-1},
\end{aligned}
$$

for $n \geq 3$, where the last equality follows from the skein relation (1) and the equality (2).

We show the second equality in Lemma 3 by induction on $n$. Let $m \geq 4$. we suppose the equality for $n<m$. Then we have

$$
\begin{aligned}
L G\left(\theta_{2}(m)\right)= & \left(T_{0}+T_{1}-T_{0} T_{1}\right) L G\left(\theta_{2}(m-1)\right)+\left(T_{0} T_{1}^{2}+T_{0}^{2} T_{1}\right) L G\left(\theta_{2}(m-2)\right) \\
& +\left(2+T_{0}+T_{1}+T_{0} T_{1}\right)(-1)^{m-1} T_{0}^{m-1} T_{1}^{m-1} \\
= & -2\left(T_{0}+T_{1}\right)^{m-2} T_{0} T_{1}+\sum_{i+j>m} a_{i, j}\left(\theta_{2}(m)\right) T_{0}^{i} T_{1}^{j} .
\end{aligned}
$$

An invariant for unordered links is that for ordered links by forgetting the order of an ordered link.

Lemma 4. For $M \in \mathcal{M}_{\infty}^{d, r}$, we have

$$
a_{i, j}(M)=0 .
$$

For $M \in \mathcal{M}_{0}^{r-1, r}$, we have

$$
a_{i, j}(M)= \begin{cases}(-1)^{r-1} & \text { if } i+j=2 r-2, \\ 0 & \text { otherwise. }\end{cases}
$$

For $M \in \mathcal{M}_{l}^{r, r}(l \geq 2)$, we have

$$
a_{i, j}(M)= \begin{cases}-2\left(\begin{array}{c}
r-2 \\
i-1
\end{array}\right) & \text { if } i+j=r=l, \\
0 & \text { if } i+j<2 r-l .\end{cases}
$$


Proof. The Links-Gould polynomial vanishes for a split link, and so is the coefficient: $a_{i, j}(M)=0$ for $M \in \mathcal{M}_{\infty}^{d, r}$.

For $M \in \mathcal{M}_{0}^{r-1, r}$, by Lemma 3 and the equality (2), we have $L G(M)=$ $L G\left(\theta_{1}\right)^{r-1}=(-1)^{r-1} T_{0}^{r-1} T_{1}^{r-1}$, which implies

$$
a_{i, j}(M)= \begin{cases}(-1)^{r-1} & \text { if } i+j=2 r-2 \\ 0 & \text { otherwise }\end{cases}
$$

For $M \in \mathcal{M}_{l}^{r, r}(l \geq 2)$, by Lemma 3 and the equality (2), we have

$$
L G(M)=2(-1)^{r-l+1}\left(T_{0}+T_{1}\right)^{l-2} T_{0}^{r-l+1} T_{1}^{r-l+1}+\sum_{s+t>2 r-l} a_{s, t}(M) T_{0}^{s} T_{1}^{t},
$$

which implies

$$
a_{i, j}(M)= \begin{cases}-2\left(\begin{array}{c}
r-2 \\
i-1
\end{array}\right) & \text { if } i+j=r=l \\
0 & \text { if } i+j<2 r-l\end{cases}
$$

Proof of Theorem 1. By the definition of the R-matrix, we have

$$
R-\left.R^{-1}\right|_{t_{1}=1+\alpha_{1} h} ^{t_{0}=1+\alpha_{0} h}=h X,
$$

for some $16 \times 16$ matrix $X$ such that $\left.X\right|_{h=0} \in M_{16}\left(\mathbb{C}\left[\alpha_{0}^{ \pm 1 / 2}, \alpha_{1}^{ \pm 1 / 2}\right]\right)$. Then, for a singular link $L$ with more than $d$ singular points $(d \geq 2)$, we have

$$
L G\left(L ; 1+\alpha_{0} h, 1+\alpha_{1} h\right)=\sum_{i+j>d} a_{i, j}(L) \alpha_{0}^{i} \alpha_{1}^{j} h^{i+j}
$$

which implies that $\sum_{i+j=d} a_{i, j}(L) \alpha_{0}^{i} \alpha_{1}^{j}$ is a finite type invariant of type $d$. This argument is essentially the same with [2]. Hence $a_{i, j}(L)$ is a finite type invariant of type $i+j$.

For $M \in \widetilde{\mathcal{M}}^{d, r}$ and an $r$-component singular link $L$, there exists an integer $m^{d, r}(M ; L)$ such that

$$
v(L)=\sum_{M \in \widetilde{\mathcal{M}}^{d, r}} m^{d, r}(M ; L) v(M),
$$

for any finite type invariant $v$ of type $d$ (cf. [2], [10], [12]). We suppose $i+j \leq r-2$. Putting $v=a_{i, j}$, by Lemma 4 , we have

$$
a_{i, j}(L)=\sum_{M \in \widetilde{\mathcal{M}}_{\infty}^{i+j, r}} m^{i+j, r}(M ; L) a_{i, j}(M)=0 .
$$


We suppose $i+j=r-1$. Putting $v=a_{i, j}$, by Lemma 4, we have

$$
a_{i, j}(L)=\sum_{M \in \widetilde{\mathcal{M}}_{\infty}^{r-1, r} \cup \mathcal{M}_{0}^{r-1, r}} m^{i+j, r}(M ; L) a_{i, j}(M)=0 .
$$

We suppose $i+j=r=2$. By [12], we have

$$
m^{2,2}\left(\theta_{1} ; L\right)=\lambda_{12}(L), \quad m^{2,2}\left(\theta_{2}(2) ; L\right)=\frac{\lambda_{12}^{2}(L)-\lambda_{12}(L)}{2} .
$$

Putting $v=a_{1,1}$, by Lemma 4 , we have

$$
\begin{aligned}
a_{1,1}(L) & =\sum_{M \in \widetilde{\mathcal{M}}_{\infty}^{2,2} \cup \mathcal{M}_{0}^{1,2} \cup \mathcal{M}_{2}^{2,2}} m^{i+j, r}(M ; L) a_{1,1}(M) \\
& =m^{2,2}\left(\theta_{1} ; L\right) a_{1,1}\left(\theta_{1}\right)+m^{2,2}\left(\theta_{2}(2) ; L\right) a_{1,1}\left(\theta_{2}(2)\right) \\
& =-\lambda_{12}^{2}(L) \\
& =-\sum_{C \in \mathcal{C}_{2}} \Lambda_{C}(L) .
\end{aligned}
$$

We suppose $i+j=r \geq 3$. Let $N \in \mathcal{M}_{r}^{r, r}$. Putting $v=\Lambda_{\Phi(N)}$, by Lemma 2, we have

$$
\Lambda_{\Phi(N)}(L)=\sum_{M \in \widetilde{\mathcal{M}}^{r, r}} m^{r, r}(M ; L) \Lambda_{\Phi(N)}(M)=m^{r, r}(N ; L) .
$$

Putting $v=a_{i, j}$, by Lemma 4 , we have

$$
\begin{aligned}
a_{i, j}(L) & =\sum_{M \in \mathcal{M}_{0}^{r-1, r}} m^{r, r}(M ; L) a_{i, j}(M)+\sum_{l=2}^{r} \sum_{M \in \mathcal{M}_{l}^{r, r}} m^{r, r}(M ; L) a_{i, j}(M) \\
& =\sum_{M \in \mathcal{M}_{r}^{r, r}}-2\left(\begin{array}{c}
r-2 \\
i-1
\end{array}\right) \Lambda_{\Phi(M)}(L) \\
& =-2\left(\begin{array}{c}
r-2 \\
i-1
\end{array}\right) \sum_{C \in \mathcal{C}_{r}} \Lambda_{C}(L) .
\end{aligned}
$$

Acknowledgment. The author would like to thank Tomotada Ohtsuki for his helpful comments. This research is partially supported by Grant-in-Aid for JSPS Research Fellowships for Young Scientists.

\section{References}

[1] J. S. Birman, New points of view in knot theory, Bull. Amer. Math. Soc. (N.S.), 28(1993), 253-287. 
[2] J. S. Birman and X. S. Lin, Knot polynomials and Vassiliev's invariants, Invent. Math., 111(1993), 225-270.

[3] D. De Wit, Automatic evaluation of the Links-Gould invariant for all prime knots of up to 10 crossings, J. Knot Theory Ramifications, 9(2000), 311-339.

[4] D. De Wit, L. H. Kauffman and J. R. Links, On the Links-Gould invariant of links, J. Knot Theory Ramifications, 8(1999), 165-199.

[5] J. Hoste, The first coefficient of the Conway polynomial, Proc. Amer. Math. Soc., 95(1985), 299-302.

[6] A. Ishii, Algebraic links and skein relations of the Links-Gould invariant, Proc. Amer. Math. Soc., 132(2004), 3741-3749.

[7] A. Ishii, The Links-Gould polynomial as a generalization of the Alexander-Conway polynomial, Pacific J. Math., 225(2006), 273-285.

[8] T. Kanenobu, Infinitely many knots with the same polynomial invariant, Proc. Amer. Math. Soc., 97(1986), 158-161.

[9] T. Kanenobu, Examples on polynomial invariants of knots and links, Math. Ann., 275(1986), 555-572.

[10] T. Kanenobu, Y. Miyazawa and A. Tani, Vassiliev link invariants of order three, J. Knot Theory Ramifications., 7(1998), 433-462.

[11] J. R. Links and M. D. Gould, Two variable link polynomials from quantum supergroups, Letters in Mathematical Physics, 26(1992), 187-198.

[12] H. Murakami, Vassiliev invariants of type two for a link, Proc. Amer. Math. Soc., 124(1996), 3889-3896.

[13] T. Ohtsuki, Quantum invariants. A study of knots, 3-manifolds, and their sets, Series on Knots and Everything, 29. World Scientific Publishing Co., Inc., River Edge, NJ, 2002.

[14] V. A. Vassiliev, Cohomology of knot spaces, Theory of singularities and its applications, 23-69, Adv. Soviet Math., 1, Amer. Math. Soc., Providence, RI, 1990. 